\title{
ANNONCES ET NOUVELLES
}

L'Association des Sociétés de philosophie de langue française (ASPLF) reunit tous les deux ans un congrès qui constitue l'une des grandes rencontres internationales de philosophes. Son Assemblee générale, réunie à poitiers le 28 août 1992, a confié à la Société romande de philosophie l'organisation de son XXVe Congrès. Celui-ci se déroulera à Lausanne (Suisse), sur le site universitaire de Dorigny (BSFH2) du jeudi 25 au dimanche 28 aout 1994.

Le thème général du Congrès sera: LA NATURE

Les communications se répartiront en huit sections:

1. Attitudes culturelles, artistiques et esthétiques face à la nature.

2. La nature dans les sciences

3. Nature et technique

4. Nature et société aspects écologiques et politiques

5. Nature, éthique et droit

6. La nature dans la philosophie antique et médiévale

7. La nature dans la philosophie moderne et contemporaine

8. La nature et les théorisations de l'humain dans les débats actuels

FRAIS DTNSCRIPTION:

\section{Avant le 1/12/1993 Dès le 1/1/1994}

Participant(e) 100 frs s. $\quad 130$ frs s.

Accompagnat (e) 50 frs s. 50 frs s.

Etudiant (e) $\quad 30$ frs s. $\quad 30$ frs s.

Le paiement de l'inscription donne droit à la remise d'un portedocuments contenant toutes les informations concernant le Congrès, à l'invitation aux réceptions et manifestations culturelles ainsi qu'à une carte de transports urbains. Il permet également de présenter une communication.

Les inscriptions seront enregistrés à la réception du bulletin et du règlement des frais d'inscription par virement bancaire. Un paiement effectue avant le 31/12/1993 donne droit au rabais indique ci-dessus. Les personnes qui paient par chèque bancaire sont priees de majorer leur chèque de 10 frs $s$. (montant moyen que la banque retient pour l'encaissement). 
Adresse bancaire: Banque Cantonale Vaudoise

UNI:, BSFH2, CH-1015 Lausanne

Compte \# 307.93.18 (Mention: XXVe Congrès ASPLF)

COMMUNICATIONS:

Les participant(e)s qui souhaitent presenter une communication sont prié(e)s de l'indiquer au moyen du bulletin ci-joint. La présentation de la communication durera 20 minutes au maximum et sera suivie d'une discussion de 10 minutes environ. Les résumés dactylographiés des communications (15-20 lignes) devront nous parvenir jusqu'au 1er mai 1994.Les textes destinés à être publiés dans les Actes du Congrès devront être remis au ier octobre 1994 et comporteront pas plus de 6 pages dactylograp[hies de 1800 signes chacune.

\section{LOCEMENT:}

L'Office du Tourisme et des Congrès de la Ville de Lausanne vous enverra une offre de logement apres votre inscription. Les prix minimaux seront de 35 frs s. par nuit et par personne, petit déjeuner inclus. Pour les hotels de la catégorie moyenne, les prix iront de 105 à 125 frs s. (150 a 170 frs s. pour deux personnes), petit déjeuner inclus. Des préréservations importantes ont été effectués dans ;es hôtels de la ville pour ces deux catégories.

\section{TRANSPORTS:}

Lausanne est facilement accessible par train ( $3 \mathrm{~h}$. de Paris en TGV) et par avion (l'aéroport de Genève-Cointrin est à $1 \mathrm{~h}$. de train par ligne directe), ainsi que par la route.

Une fois sur place, il est preférable de se servir des transports publics. Une ligne de train rapide (TSOL) relie le centre de la ville de Lausanne au campus de Dorigny. Une carte de transport urbain sera offert lors de leur enregistrement aux congressistes qui le souhaitent.

\section{DEROULEMENT}

Le Congrès débutera le jeudi 26 aoât 1994 à $20 \mathrm{~h}$. Les congressistes seront accueilli(e)s dès $16.30 \mathrm{~h}$. L'Assemblee générale de l'ASPLF réunira les président(e)s des Sociétés membres le 26 aout de 18 a $20 \mathrm{~h}$. La clôture officielle aura lieu le 28 août à $17.30 \mathrm{~h}$. 